\title{
Complex Situation Recognition on the Basis of Neural Networks in Shipboard Intelligence System
}

\author{
Yu. Nechaev, A. Degtyarev, I. Kiryukhin \\ Institute for High Performance Computing and Data Bases, \\ 120 Fontanka, 198005 St.Petersburg, Russia \\ $\{$ int, deg,ilia\}@in.csa.ru
}

\begin{abstract}
The problem of complex dynamic object (DO) state identification in extreme situation is considered. Analysis is carried out on the basis of selforganising artificial neural network (ANN). Compression of the measuring information about object dynamics is achieved by means of cognitive structures. Identification procedure is realised with the help of inference in intelligence system (IS) of unsinkability monitoring of ships and marine vehicles.
\end{abstract}

\section{Introduction}

One of the most complicated problems in estimation and forecast of DO behaviour in extreme conditions is situation identification. Critical obstacles we have especially for floating DO at holing and compartments flooding. In these conditions there are continuous modification of DO state and of factors defining its interaction with environment.

The authors use several approaches for this important practical problem solution $[5,6]$. Developed inferential mechanism includes various firmware procedures (statistical analysis, researches of phase portraits, mathematical modelling). However, in conditions of uncertainty and incompleteness of initial information such approach does not always ensure satisfactory results. Searching of more effective situation identification methods has resulted in using ANN theory $[2,6-8,10]$. Neural network approach is successfully applied by the authors in problems concerning with knowledge formalisation and multicriteria optimisation in IS of marine DO control.

Novelty of considered approach consists in the following:

- Processing of measuring information at input in ANN is realised by cognitive graphics methods;

- Improvement of identification effectiveness is achieved on the basis of alternatives analysis by comparison of results obtained on multilayer ANN models and Kohonen's self-organising maps;

- Results of identification are used as firmware procedure of fuzzy inferential mechanism.

In the paper ANN application is considered in framework of competing computation technology in parallel information processing on multiprocessor systems [7]. Principles of this technology allow to organise selection of the most effective 
algorithm for measured information processing in real-time IS during alternatives analysis. As a result, reliability of assessments of characteristics of DO-environment interaction in complex situations is increased. Strategy of alternative selection in framework of competition principle is realised in the following way:

- generation of alternatives with the help of ANN basis and traditional algorithms of DO and environment characteristics control;

- alternatives analysis and choice of the best model;

- development of preferable alternative and result estimation.

Proposed conception is important both on stages of IS design and testing, and in operation. Comparison of different algorithms for obtaining dynamic characteristics of the control object and environment with modelling results based on ANN allows to estimate merits and demerits of used methods and to choose rational ways for information basis forming in production inference rules.

Another important advantage of developed technology consists in collecting data about ANN algorithms for solution of poor formalised problems. These data ensure formation of library of applied neuromathematics algorithms for realisation on neurocomputers which are very perspective high performance computational tools. The main advantage of ANN related with nonlinear information transformation allows to realise complicated algorithms of decision support for DO control in fuzzy environment. By collecting such information it is possible to organise learning process in such a way that the best solution will adopted in conditions of incompleteness and uncertainty of initial information.

\section{Conceptual model}

Let us consider method of automated synthesis of structure and determination of ANN parameters. Problem solution of DO state identification is carried out in accordance with realisation of parameters complex formed on the basis of information from measuring system. It is supposed that diagnosis areas in characteristics space are noncompact, i.e. nonempty intersection of the appropriate areas takes place. The concept and approach provide construction of ANN structure adequately describing characteristics of discovered situation. Sequence of information transformation determines solution of the following tasks:

- compression of information and its representation as convenient input for ANN perception;

- choice of ANN structure in conditions of continuous modification of object dynamics and environment;

- learning of ANN in accordance with sequence of extreme situations appropriating to classical flooding cascs of the unsinkability theory.

Considered emergency conditions are represented by various areas of the diagnosis in attribute space. Correct recognition of situations by the developed classification rule can be reached on the basis of work [5]. ANN ability of selflearning and its parallelism allow to realise analysis and forecast of emergency situation in real time.

ANN is represented as "black box" at problem formalisation. Exterior information acts on input of such "black box". Desirable signal is realised on the output as vector 
of situation characteristics. As preliminary investigations have shown ANN is capable to completely transform its own behaviour at object dynamics and environment modification.

Algorithm of information transformation at realisation of inference mechanism is shown on the basis of production model $[1,2,5,6]$ :

$$
\begin{aligned}
& P_{i}:\left\langle\text { if } X_{i, q} \in \Phi_{0} \& X_{j, k}^{*} \& X_{j, k}^{* *} \in \Phi, \text { then } Y_{i}, \text { else } Z_{i}\right\rangle \\
& (i=1, \ldots, N ; q=1, \ldots, S ; j=1, \ldots, m, k=1, \ldots, n),
\end{aligned}
$$

where $P_{i}$ is the name of production; $X_{i, q}$ is the classified situation; $X_{j, k}^{*}$ is the vector of measurements; $X_{j, k}^{* *}$ is the vector of modelling parameters; $\Phi_{0}$ is the area of standard situations; $\Phi$ is the area of admissible values of situation characteristics; $Y_{i}$ is the consequent of production; $Z_{\xi}$ is the alternative choice; $N$ is the number of productions; $S$ is the number of standard situations classified by ANN; $m, n$ are the numbers of parameters characterising vectors of measurements and modelling.

Thus various firmware procedures are used at interpretation of production kernel in antecedent of implication. Engineering process of IS construction assumes alternation of steps of situations modelling and decision making synthesis. Solution is achieved with the help of ANN and cognitive structures [11]. Check of extreme situation recognition reliability is carried out by methods of simulation modelling ensuring estimation and forecast of object (ship) dynamics with flooded compartment recognised by ANN:

$$
F_{t, m} \in\left\{F_{M}, P(X)\right\}(t=\overline{1, T})
$$

where $F_{M}$ is the operator mapping situations set $\{\mathrm{X}\}$ in behaviour set $\{\mathrm{Y}\}$ of local model $F_{m} ; t$ is considered time moment; $P(X)$ is the probability distribution describing set of input data acting from measuring system.

Adequacy condition is determined as

$$
Y \in S \forall c \in C_{m},
$$

where $S$ is the area bounding behaviour of model; $C_{m}$ is the purposes of modelling.

Developed formalised knowledge system allows to supply monitoring and forecast of extreme situation development.

\section{Cognitive structures}

Solution of recognition problem by using cognitive paradigm [11] was carried out for extreme situation connected with estimation of damaged ship dynamics on waves. Such approach permits to obtain occurrence and development of oscillatory regime of damaged ship depending on character of flooding and level of external excitation. Searching of concrete mapping model was carried out with the help of cognitive spiral. It permits "to compress" initial information about non-linear rolling with a 
casual changing of periods. Construction of cognitive spiral on random function leads to periods "justification" and transformation of them to one value by means of affine compression or stretching of researched function intervals up to given size.

Cognitive spiral, as alternative to a phase portrait, contains more information, which it is possible to select visually orienting on image structure. Breadth of bands in spiral, its saturation by colour and frequency of changing of band of one colour by band of other colour can be referred to such information. Asymmetry of upper and lower parts of cognitive spiral and distribution of colour on angles close to 0 and 180 degrees can also play important role.

Considered recognition problem is connected with classification of "difficult to separate" typical cases of flooding among five classical situations defined at estimation of damaged ship dynamics [5,9]. These situations are characterised by variation of righting component of mathematical model of damaged ship rolling. Among them it is necessary to mark out second, third and fourth cases. The first case is trivial enough: lack of static heel at symmetric flooding and positive initial metacentric height. In other emergency states ship always has heel on a side. It allows to easily pick out first case without using complicated inference procedures. As regards to the fifth case it is usually considered as subset of fourth case. Similar pictures of oscillation are typical for this case.

Let us describe specific features of researched cases:

- Second typical case. Cognitive structures have intensive dark bands. These bands are divided by smaller in breadth light bands. Spiral is symmetric relative upper and lower parts and has steady light area at angles close to 0 and 180 degrees.

- Third typical case. Main difference of this cognitive structure is asymmetry of upper and lower parts of spiral. At angles close to 0 and 180 degree there no neutral light bands. Here there is no clear changing of bands, as in second case, dominance of light hues in lower part of spiral occurs.

- Fourth typical case. Cognitive spiral is symmetric and has very few light bands (actually there is alternation of more and less dark bands).

Examples of cognitive structures for cases 2 and 3 are shown in the fig. 1 .

Analysis of cognitive structures permits to mark out information for recognition. This problem is solved by determination of rational compromise between two factors. On the one hand, it is necessary to have maximum information for obtaining best classification possible. On the other hand, it is important to reduce information volume at entrance in ANN in connection with reduction of learning time and computer resources. Rational solution of this problem is obtained by selection of characteristic radial slits on some angles in cognitive spiral. Typical were slits at angles of 90 and 270 degrees representing frequency of bands change, their breadth and colour saturation. For qualitative comparison it is possible to use angles near 0 and 180 degrees, for example, $\pm 10,170$ or 190 degrees.

Searching of rational ways of data transformation resulted in reviewing of no realisations, but of appropriate correlation functions or spectral densities constructed at quasistationarity intervals. As a result the constant number of input neurones is established, and ANN structure is determined. At that we have freedom in choice of realisation length depending on conditions of IS functioning reliability in extreme situations estimation. For recognition accuracy improvement and time reduction of ANN learning, moving average is excluded from used realisations. Correlation function for the "cleared" data is calculated. 

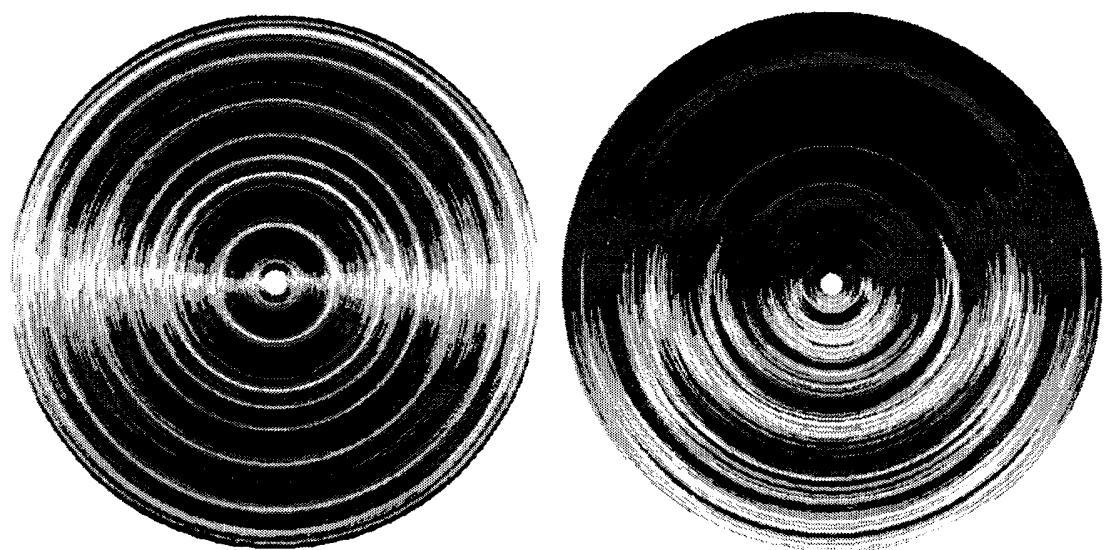

Fig. 1. Cognitive structure for flooding cases 2 (left image) and 3 (right image).

Comparison of spectral densities for considered flooding cases testifies about possibility to use them in classification problem. However correlation functions have great visual distinction in situations $2-4$, therefore using of correlation functions are more preferable.

\section{Multilayer neural network topology}

ANN represents series connection of $\mathrm{K}$ layers of formal neurones in a view of coneshaped configuration (fig. 3 ). Each layer contains $n_{i}(i=1, \ldots, K)$ neurones, whose number regular by decreases with increasing of layer number. Each neuron or base processor element (BPE) is characterised by series connection of two components: linear converter (summator) of multidimensional input vector $r_{j}$ into one-dimensional summator output vector $S$ with weight coefficients $W_{i j}\left(i=1, \ldots, K ; j=1, \ldots, n_{i}\right)$ and non-linear transformation of summator output $S$ into output signal

$$
q=f\left(\sum_{j=1}^{n} w_{j} r_{j}\right)=f(S) .
$$

Thus, multilayer ANN structure ensures complicated non-linear transformation of input vector $r=\left\{r_{j}\right\}$ depending on vectors of weight coefficients $W=\left\{w_{i j}\right\}$ and nonlinear activation functions $f(\cdot)$.

Relation between input and output of ANN is determined by the following nonlinear recurrent equation

$$
\begin{aligned}
& Y=q^{(k)}=f^{(k)}\left(W_{0}^{(k)}+W_{1}^{(k)} f^{(k-1)}\left(W_{0}^{(k-1)}+W_{1}^{(k-1)} f^{(k-2)}\left(\ldots W_{0}^{(l)}+W_{1}^{(l)} f^{(l-1)} \times\right.\right.\right. \\
& \left.\left.\left.\left(W_{0}^{(l-1)}+W_{1}^{(l-1)} f^{(l-2)} \times\left(\ldots W_{0}^{(2)}+W_{1}^{(2)} f^{(1)}\left(W_{1}^{(1)}+W_{1}^{(1)} q^{(0)}\right) . .\right)\right) \ldots\right)\right)\right)=F(r)
\end{aligned}
$$


where $F(r)$ is a non-linear function.

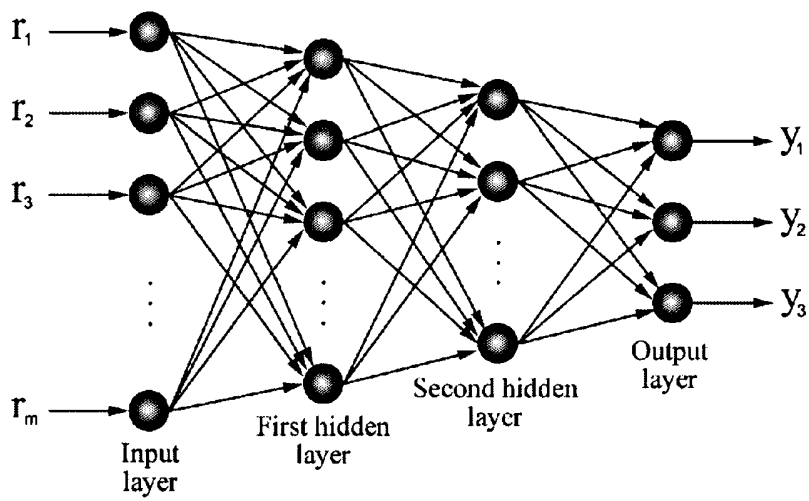

Fig. 2. Multilayer neural network

Both correlation functions and spectral densities of "cleared" slits of cognitive spirals are used as input information for recognition. In spite of so different input data ANN has identical structure. Input layer has the number of neurones corresponding to the number of points in given image. Output layer has 3 neurones (on one neuron in each classified case). Activation functions are chosen as follows: either sigmoid function is used for all three layer, or first two hidden layers have sigmoid function, and third, output layer, uses modified linear function.

Configuration and basic characteristics of ANN are shown in Table 1.

Table 1. ANN structure and basic characteristics

\begin{tabular}{ll}
\hline Characteristics & Values and descriptions \\
\hline Input layer & 16 neurones \\
First hidden layer & 12 neurones, sigmoid activation function \\
Second hidden layer & 8 neurones, sigmoid activation function \\
Output layer & 3 neurones, modified linear activation function \\
The number of images & 45 \\
Learning coefficient & 0.1 \\
Learning accuracy, $\%$ & 100 \\
Number of learning steps & 400 \\
\hline
\end{tabular}

During ANN learning BPE weight coefficients are tuned, insuring solution of extreme situations recognition. "Back propagation" iterative procedure was used for determination of $W_{i j}(k)$ values:

$$
W_{i j}^{(r)}(k+1)=W_{i j}^{(r)}(k)+\mu\left(-\nabla_{i j}^{(r)}(k)\right), \quad r=1,2, \ldots
$$

where $k$ is the iteration number, which corresponds to input realisation $X^{0}(k)$ chosen by random way from population of input data; $\mu$ is the constant defining convergence of iterative procedure $(6) ; \nabla_{i j}(k)$ is the gradient computed by the formula

$$
\nabla_{i j}^{r}(k)=\partial \varepsilon_{u}^{2}(k) / \partial W_{i j}^{(k)}(k) .
$$




\section{Competing model - Kohonen's self-organising map}

As has been shown in investigation [6] some problems in classification of dynamically varying situations can be observed at practical realisation of multilayer ANN. Phase space topology in such situations is kept stable only during limited time intervals. It results in unstable work of ANN. Competitive Kohonen's model is used for this problem solution [4].

Elimination of possibility of multiple-valued solution in Kohonen's map (fig.3) is ensured by mean of additional structure insertion permitting organise recognition procedure with the help of mechanism called "competition". Extreme form of competition in group of neurones is principle "winner takes all".

Kohonen's learning algorithm. Algorithm uses tuning neuron through its own weights modification and new weight vector formation. During self-organising process cluster mesh, whose weight vector "gains" input image (closest to input image), is chosen by the winner. This mesh and the nearest neighbours change their own weights. At that learning velocity was accepted as slowly decreasing time function.

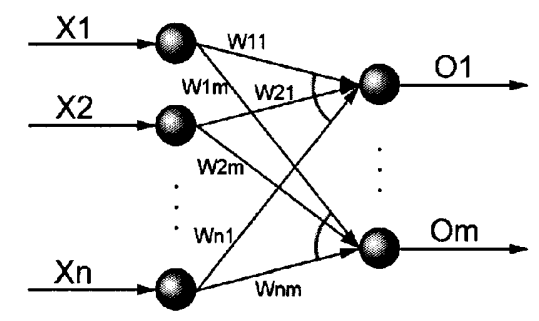

Fig. 3. Kohonen's self-organising map.

Searching of effective solutions has resulted in using of uncertainty at choice of weight coefficients, which are necessary for changing during learning. Standard learning method allows to change only gained neuron weights. In fuzzy approach all weights are varying in accordance with output neurones activation functions which are calculated for pattern given on ANN input. Such approach allows adjust weight matrix and divide space of objects into classes more precisely.

In considered classification problem uncertainty was used not only in learning, but also in inference. If we introduce normalisation of output neurones activity, we shall receive probabilities of acceptance of hypothesis about membership of image to concrete class. Analogy with fuzzy logic allows to consider Kohonen's selforganising map as a system for object activation function construction. Here competition for ANN organisation offered by Kohonen is retained, but it is less obvious at the fuzzy approach. Results of investigations show, that additional restrictions are imposed on initial conditions at learning with uncertainty elements. In particular, it is impossible to set identical columns in weight matrix. At lack of distinctions between weight coefficients their modification at the same activation of output neurones will happen quite equally. Therefore weight vectors could be tuned on the same class. 


\section{ANN weight coefficients adjustment by means of genetic algorithm}

As competitive strategy of ANN learning (both multilayer ANN and Kohonen's selforganising map) genetic algorithm (GA) also has been used [3]. Let us note GA features applied for global optimum searching in space of weight coefficients:

- co-ordinates of weight coefficients were determined in range $[-2 ;+2]$, and range was divided into 255 parts;

- chromosome coding was carried out with the help of Gray code;

- chromosome suitability was calculated through the goal function $Q$.

For multilayer ANN function $Q$ looks like

$$
Q=\sum_{k, j}\left(Y_{j}^{k}-D_{j}^{k}\right)^{2} \rightarrow \min ,
$$

where $Y_{j}^{k}$ is the signal from $j$-th neuron of output layer at the $\mathrm{k}$-th learning example, $D_{k}^{j}$ is the desired signal from $\mathrm{j}$-th neuron of output layer.

For Kohonen's self-organising map goal function $Q$ is

$$
Q=\sum_{k}\left(\min _{j} \sum_{i}\left(W_{i}^{j}-X_{i}^{k}\right)^{2}\right) \rightarrow \min ,
$$

where $W^{j}$ is the vector adjusted to the gravity centre of $\mathrm{j}$-th cluster, $X^{k}$ is the $\mathrm{k}$-th input vector.

Population size was selected a priori for practical reasons. As experiments have shown, GA demonstrates satisfactory results both in small and in large population sizes. With a view to decrease evolution time population size of 10 chromosomes was set up for our problem. In every evolution step one-point crossover, mutation, cyclic shift and selection proportionally suitability were realised. Probabilities of genetic operators are shown in Table 2.

Table 2. Genetic operators and there probabilities

\begin{tabular}{|l|l|}
\hline Genetic operator & Probability \\
\hline One-point crossover & 1.00 \\
\hline Chromosome mutation & 0.10 \\
\hline Bit inversion in chromosome mutation & 0.10 \\
\hline Cyclic shift & 0.05 \\
\hline
\end{tabular}

Learning with the help of GA took more time than using of standard methods of networks learning. It is necessary to note that ANN with GA adjusted coefficients recognises cognitive spirals not worse than ANN, trained by standard method. GA advantages are:

- algorithm is not strongly linked to concrete activation function, as "back propagation" method (function can be non-differentiable and even discontinuous);

- searching in all weight coefficients space; 
- this is stochastic searching.

Thus, GA is more universal algorithm of global optimum search, which, besides, is easily realised on parallel computing architecture.

\section{Results of experiments}

Investigation of possibility to use obtained results and efficiency of ANN was carried out. For these purposes practical classification problem of real IS was considered. Research included generation of real sea waves and damaged ship - environment interaction modelling with the help of specially developed tool for IS testing. This tool is complemented by subroutines of cognitive structures construction and processing and ANN learning. During research qualitative comparison of classification results for "short" and "long" realisations of non-linear stochastic processes was also carried out. These processes characterise damaged ship rolling on irregular waves.

Interesting results were obtained at the analysis of activation function influence on speed of ANN weights convergence. So, for example, semilinear (modified linear) activation functions

$$
Y_{i}=\left\{\begin{array}{l}
0, S_{j}<0 ; \\
k S_{j}, 0 \leq S_{j} \leq 1 / k ; \\
1, S_{j}>1 / k .
\end{array}\right.
$$

could not be always used on all layers. At the same time, such function proves to be suitable for output layer where hypothesis about type of flooding is finally formed. Here semilinear function is limited and quickly reaches maximal values. Sigmoid function fits well for all other network layers (input and hidden). Using of such function on output layer only increases learning time and practically does not improve classification accuracy.

Research of realisation length influence is important for reduction of learning time at network adaptation in case when extreme situation development occurs during short time interval. In this case it is necessary to estimate situation very quickly and to take safety measures for ship survivability.

In using Kohonen's self-organising maps the same correlation functions were given on neural network input as on multilayer ANN input. It allowed to compare results and to estimate efficiency of used neural networks. As results of the research show, Kohonen's self-organising maps in some cases have certain advantages as compared to multilayer networks. These advantages provide certain split of situations and reference recognised object only for one concrete class. Besides, unsupervised learning has also other advantages. So, for example, in the second typical flooding case ship dynamics on irregular waves in conditions of limited excitations could be close to the third typical case. At supervised learning such situation results in occurrence of learning mistake: some rolling realisations of damaged ship could be incorrectly classified. But, if we use unsupervised learning, such variant joins class of realisations of the third typical case. As a result it is not possible to avoid learning mistakes. Way out from such situation is joint classification on the basis of considered ANN. It allows to essentially increase efficiency of recognition process. 


\section{Conclusions}

Concept of on-board IS design as a system of parallel operation provides ANN using not only as general method for analysis and dynamic measuring data interpretation in DO control systems, but also as competing technology for data processing in complex situations analysis. As a result of alternatives analysis technology is preferable if it ensures more reliable assessment of researched situation in accordance with adopted discriminating rule. Just this technology will be developed in future during solution of current problem of analysis and interpretation of dynamic measuring data.

Researches carried out show great opportunities of using ANN in problems of extreme situations identification when DO-environment interaction conditions are continuously changing. Quality of ANN functioning is achieved by means of variation of operational principles and formation of criteria, which ensure flexibility and ability to external conditions adaptation. As a result perspectives of development of information technologies for important practical problems solution are opening. In this paper we have discussed application of such new information technologies to ensuring ships' unsinkability.

The research is supported by grant of RFBR N 00-07-90227.

\section{References}

1. Averkin A.A. Soft computing is base of new information technologies // Proc. of 5-th national conference on artificial intelligence with international participation, Kazan, 1996, vol.2, pp.237-239. (in Russian)

2. Bogdanov A., Degtyarev A., Nechaev Yu. Fuzzy logic basis in high performance decision support systems. Computational Science - ICCS 2001, LNCS 2074, Springer, 2001, pp.966-975

3. Goldberg D. E. Genetic algorithms in search, optimisation, and machine learning. Reading, MA: Addison-Wesley. 1989.

4. Kohonen T. Self-Organizing Maps. Springer-Verlag, Heidelberg, 1995

5. Nechaev Yu.I., Degtyarev A.B., Boukhanovsky A.V. Identification of extreme situation in fuzzy condition. // Proc. of international conference on fuzzy computing and measurement SCM-98. St. Petersburg, 1998, vol.1.pp.85-88. (in Russian)

6. Nechaev Yu.I., Siek Yu.L., Vasyunin D.A. Neural network technology in the intelligence system of marine technics. // Proc. of $6^{\text {th }}$ national conference on artificial intelligence with international participation, Puschino, 1998, vol.2, pp.361-368. (in Russian)

7. Nechaev Yu.I. Neural network technologies in real time intelligence systems. //Proc. of $4^{\text {th }}$ National conference "Neuroinformatics 2002", Moscow, 2002, v.1, pp.1-55 (in Russian)

8. Wassermam F. Neurocomputer technics. Theory and practice. - Moscow: Mir, 1992.

9. Ship Theory Handbook. Ed. by Y.I.Voitkounski In 3 volumes, Vol.2. Statics of Ship, Ship Motions, Leningrad. "Sudostroenie", 1985. (in Russian)

10. Zadeh L. Fuzzy logic, neural networks and soft computing. //Communication of the ACM, 1994, vol.37, N3, pp.77-84.

11. Zenkin A.A. Cognitive computer graphics. - Moscow: Nauka, 1991. (in Russian) 